\title{
Haemodynamic Characteristics And Factors Influencing The Head-Up Tilt Test In Patients With Orthostatic Hypotension
}

\section{Wang Jiayu}

Capital Medical University

\section{Wu Yueyang}

Capital Medical University

\section{Wang Yongjun}

Capital Medical University

\section{Zhao Xingquan}

Capital Medical University

\section{Wang Yilong}

Capital Medical University

\section{Li Yinghui}

China Astronaut Research and Training Center

Wu Bin

China Astronaut Research and Training Center

Wang Linjie

China Astronaut Research and Training Center

Qu Lina

China Astronaut Research and Training Center

\section{Li Zhaoxia}

Capital Medical University

\section{Yang Xiaomeng}

Capital Medical University

\section{Andre Aubert}

University Hospital Gasthuisberg

Liu Jiexin ( $\sigma$ jiexinliu@hotmail.com )

Capital Medical University

\section{Research Article}

Keywords: Orthostatic hypotension, syncope, head-up tilt test, autonomic neural regulation 
Posted Date: May 26th, 2021

DOI: https://doi.org/10.21203/rs.3.rs-506163/v1

License: (c) (i) This work is licensed under a Creative Commons Attribution 4.0 International License. Read Full License 


\section{Abstract}

Background: Failure to activate autonomic neural and hormonal reflex mechanisms could result in orthostatic hypotension. Patients with orthostatic hypotension often experience symptoms of cerebral hypoperfusion, including syncope. The purpose of this study was to investigate the haemodynamic characteristics of patients with orthostatic hypotension and the factors affecting blood pressure regulation.

Methods: This retrospective study enrolled 45 patients who were diagnosed with typical orthostatic hypotension by the head-up tilt test at Beijing Tiantan Hospital, Capital Medical University from June 2019 to December 2020. Changes in haemodynamic parameters in these patients were compared in the supine and tilted upright positions.

Results: For all patients, haemodynamic parameters in the supine and tilted upright positions were compared, and systolic blood pressure, diastolic blood pressure, mean blood pressure, cardiac output and stroke volume were all significantly decreased per minute. Heart rate significantly increased per minute, but systemic vascular resistance did not significantly change. The absolute value, percentage and rate of increases in heart rate and the absolute value, percentage and rate of decreases in stroke volume from the supine to the titled upright position were significantly lower in patients 60 years and older than in patients under 60 years old. Linear regression analyses found that the changes in heart rate, stroke volume and systemic vascular resistance were related to changes in systolic blood pressure, while the changes in stroke volume and systemic vascular resistance had more important effects on systolic blood pressure.

Conclusions: In patients with orthostatic hypotension, we observed no significant increase in systemic vascular resistance from the supine position to the tilted upright position. Nevertheless, an increase in systemic vascular resistance had a significant impact on the maintenance of systolic blood pressure. In addition, in orthostatic hypotension patients younger than 60 years of age, heart rate increased while stroke volume decreased significantly after tilting upright compared with orthostatic hypotension patients older than 60 years of age and older.

\section{Background}

Orthostatic hypotension $(\mathrm{OH})$ is a key manifestation of autonomic dysfunction that is observed when cardiovascular autoregulation mechanisms cannot compensate for the decrease in returned blood volume from the supine to tilted upright position ${ }^{1,2}$. The aetiology of $\mathrm{OH}$ is multifactorial and divided into non-neurogenic and neurogenic causes ${ }^{3}$. $\mathrm{OH}$ is the second most common aetiology of syncope, and it occurs in approximately $15 \%$ of syncope presentations ${ }^{4-6}$.

Patients with $\mathrm{OH}$ often experience symptoms of cerebral hypoperfusion, including dizziness, fatigue, head ache and even syncope ${ }^{6}$. Therefore, $\mathrm{OH}$ contributes substantially to the risk of falls and disability 
and seriously impacts quality of life. The incidence of $\mathrm{OH}$ increases with age, which affects $5-16 \%$ of the middle-aged and elderly community and over $50 \%$ of the elderly patients in nursing homes and geriatric wards ${ }^{7}$. It frequently affects older people and patients who have neurodegenerative disease, diabetes or hypertension ${ }^{8}$. Unfortunately, $\mathrm{OH}$ is often unrecognized or misdiagnosed and may be an overlooked factor associated with increased cardiovascular morbidity and all-cause mortality ${ }^{7,9}$.

Given the ageing population worldwide, the potential impact of $\mathrm{OH}$-related hospitalizations and the depletion of medical resources, it is critical to identify groups of patients at risk for $\mathrm{OH}$ and to use appropriate methods to treat $\mathrm{OH}$. Consequently, the purpose of the current study was to investigate the haemodynamic characteristics of patients with $\mathrm{OH}$ and the factors affecting blood pressure regulation.

\section{Methods}

Study population

This retrospective study enrolled patients attending the syncope clinic due to syncope or dizziness who were diagnosed with $\mathrm{OH}$ by the head-up tilt test at the Beijing Tiantan Hospital, Capital Medical University from June 2019 to December 2020. According to the definition and classification of $\mathrm{OH}$, our study focused on typical $\mathrm{OH}$ and excluded initial and delayed subsets of $\mathrm{OH}$. The study protocol conformed to the ethical guidelines of the Declaration of Helsinki and was approved by the Ethics Committee of the Beijing Tiantan Hospital. The methods were strictly carried out in accordance with the approved guidelines. Confirm informed consent of all subjects and, if subject is under 18 years of age, obtain informed consent from parent and/or legal guardian.

Tilt-test protocol and measurements

Tests were performed between 9:00 AM and 1:00 PM in a temperature-controlled room $\left(23^{\circ} \mathrm{C}\right)$. For 2 hours prior to the test, no medications were taken by the patient, while a small amount of water was allowed. A manually operated tilt table with a footboard was used. According to the Italian protocol, the tilt test started with 5 minutes of supine rest, followed by 20 minutes of head-up tilt $\left(70^{\circ}\right)$ in the unmedicated phase. If no syncope or presyncope developed, nitroglycerin was administered sublingually $(0.25 \mathrm{mg})$ for an additional 15 minutes tilt duration. An impending syncope was aborted by means of tilt back or by countermaneuvers such as leg crossing to prevent loss of consciousness ${ }^{10}$. Blood pressure was measured continuously and noninvasively using the Finapres Model 1 (Finapres Medical System B.V., the Netherlands).

Diagnostic criteria ${ }^{3,11,12}$

Typical $\mathrm{OH}$ was defined as a sustained reduction of either systolic blood pressure of at least $20 \mathrm{mmHg}$ or diastolic blood pressure of $10 \mathrm{mmHg}$ within 3 minutes of head-up tilt to at least $70^{\circ}$ on a tilt table. Initial $\mathrm{OH}$ was defined as a transient blood pressure decrease $(>40 \mathrm{mmHg}$ systolic blood pressure and/or $>20$ 
$\mathrm{mmHg}$ diastolic blood pressure) within 15 seconds of standing. Some patients with symptomatic $\mathrm{OH}$ occurring beyond 3 minutes of standing were defined as having delayed $\mathrm{OH}$.

Haemodynamic analysis

Haemodynamic data were collected using a noninvasive continuous beat-to-beat blood pressure monitor. The finger clip was placed on the second knuckle of the middle finger of the right hand, and the height sensor was fixed at the level of the finger clip and the patient's heart.

The continuous blood pressure data were corrected by using the built-in upper arm cuff blood pressure metre before the head-up tilt test began.

Beat-to-beat systolic and diastolic blood pressures (SBP and DBP) were derived from the arterial pressure waveform. Mean arterial pressure (MAP) was computed as true integral of the arterial pressure wave over one beat divided by the corresponding beat interval. Heart rate (HR) was computed as the inverse of the interbeat interval (IBI) and expressed as beats per minute. Beat-to-beat changes in stroke volume (SV) were estimated by modelling the flow from the finger arterial pressure waveform (Model Flow, Finapres Medical System). The patient's sex, age, height and weight were included in the model to determine Model Flow parameters using previously established equations. Cardiac output (CO) was computed by the product of SV and HR. Systemic vascular resistance (SVR) was obtained through a self-adapting process in which an initial value was assumed for the first beat. The ratio of MAP to $C O$ for this first beat defined a new resistance value that was used in the model for the next beat ${ }^{13-15}$.

The mean value of beat-to beat data 5 minutes before the head-up tilt test was calculated as the haemodynamic parameters in the supine phase. If the patient did not experience presyncope ${ }^{12}$, a significant decrease in blood pressure (SBP $\leq 80 \mathrm{mmHg}$ or DBP $\leq 50 \mathrm{mmHg}$ ) or bradycardia (sinus HR $\leq$ 40 beats per minute $>10$ seconds, with or without sinus arrest $\geq 3$ seconds) within 3 minutes of $70^{\circ}$ passive tilt, the mean values of haemodynamic parameters were calculated per minute of tilted upright position (1st minute, 2nd minute and 3rd minute). If the patient experienced presyncope, a significant decrease in blood pressure or bradycardia within 3 minutes of $70^{\circ}$ passive tilt, the head-up tilt test was terminated, and the mean values were calculated as the haemodynamic parameters per minute in the actual tilted upright time.

After the patient was tilted upright, the minimum values of SBP, DBP and MBP were defined as SBPmin, DBPmin, and MBPmin, respectively; the minimum values of SV and CO were defined as SVmin and Comin, respectively; and the maximum values of HR and SVR were defined as HRmax and SVRmax, respectively. The time from patient passive tilting upright to the onset of SBPmin was defined as the Time to SBPmin.

Rate of change of haemodynamic parameters in the tilted upright position 
In this study, the second derivative algorithm was used to calculate slopes. Slopes were used to evaluate the rate of haemodynamic parameters after patients tilted upright. The rates of changes in haemodynamic parameters every minute were calculated for 3 minutes after patients were tilted and upright. The rates denoted by $\mathrm{d} / \mathrm{dt}$, and the asterisk represented haemodynamic parameters.

Statistical analysis

Statistical analyses were performed using SPSS for Windows, version 23.0. Normally distributed continuous variables were expressed as the mean \pm standard deviation and compared using Student's ttest. Nonnormally distributed continuous variables were expressed as medians (upper quartile, lower quartile) and compared using the rank sum test. Normality was tested using the Shapiro-Wilk test. Categorical variables were expressed as percentages, and the $\chi 2$ test or Fisher's exact test was used for comparisons between the two groups. Repeated measures ANOVA was used to compare changes in haemodynamic parameters between the supine and tilted upright positions. Linear regression analyses were performed to identify related variables and degrees of influence on the decrease in SBP. All statistical tests were run as two-sided tests. For all tests, $\mathrm{P}<0.05$ was considered to be statistically significant.

\section{Results}

Study population

The study included 93 patients diagnosed with $\mathrm{OH}$ by the head-up tilt test in the syncope clinic of Beijing Tiantan Hospital, Capital Medical University from June 2019 to December 2020. Ten patients who were diagnosed with delayed $\mathrm{OH}$ and 38 patients with initial $\mathrm{OH}$ were excluded. Finally, a total of 45 patients were enrolled in our retrospective study, including 36 males and 9 females. Patients were divided into two groups according to age; the first group was composed of 23 patients who were 60 years old or older, and the second group was composed of 22 patients who were under 60 years old. There were no significant differences in sex or incidence of diabetes mellitus, hyperlipidaemia, coronary heart disease, cerebral vascular disease or peripheral vascular disease between the two groups. In the 60 and older group, the number of patients with hypertension was significantly higher than that in the younger 60 group (12/23, $52.17 \%$ vs. $5 / 22,22.72 \%, p=0.042$ ).

Haemodynamic analysis

For all patients, haemodynamic parameters in the supine and tilted upright positions were compared, and SBP, DBP, MBP, CO and SV significantly decreased per minute. HR significantly increased per minute, but SVR did not change (Table 1 ). 
Table 1

Comparison of haemodynamic parameters between supine and titled upright positions

\begin{tabular}{|c|c|c|c|c|c|c|c|}
\hline Variables & Supine & $\begin{array}{l}1 \text { st } \\
\text { minute }\end{array}$ & $\begin{array}{l}\text { 2nd } \\
\text { minute }\end{array}$ & $\begin{array}{l}\text { 3rd } \\
\text { minute }\end{array}$ & $\underset{\text { value* }}{p}$ & $\underset{\text { value** }}{p}$ & value $^{\boldsymbol{p}}$ \\
\hline $\mathrm{SBP}(\mathrm{mmHg})$ & $\begin{array}{l}136.77 \pm \\
23.02\end{array}$ & $\begin{array}{l}115.49 \pm \\
18.25\end{array}$ & $\begin{array}{l}105.09 \pm \\
19.21\end{array}$ & $\begin{array}{l}104.80 \pm \\
22.10\end{array}$ & $\hat{0}_{0.001}$ & $<0.001$ & 1.000 \\
\hline $\mathrm{DBP}(\mathrm{mmHg})$ & $\begin{array}{l}69.85 \pm \\
10.23\end{array}$ & $\begin{array}{l}61.81 \pm \\
10.43\end{array}$ & $\begin{array}{l}60.08 \pm \\
11.38\end{array}$ & $\begin{array}{l}60.01 \pm \\
12.65\end{array}$ & $\begin{array}{l}<.001 \\
0.00\end{array}$ & 0.148 & 1.000 \\
\hline $\mathrm{MBP}(\mathrm{mmHg})$ & $\begin{array}{l}94.90 \pm \\
13.91\end{array}$ & $\begin{array}{l}80.55 \pm \\
12.52\end{array}$ & $\begin{array}{l}76.30 \pm \\
13.78\end{array}$ & $\begin{array}{l}76.06 \pm \\
15.75\end{array}$ & $\begin{array}{l}< \\
0.001\end{array}$ & 0.002 & 0.999 \\
\hline $\begin{array}{l}\text { HR } \\
\text { (beats/min) }\end{array}$ & $\begin{array}{l}68.14 \pm \\
10.52\end{array}$ & $\begin{array}{l}74.75 \pm \\
15.74\end{array}$ & $\begin{array}{l}79.85 \pm \\
17.64\end{array}$ & $\begin{array}{l}80.22 \pm \\
18.36\end{array}$ & $\begin{array}{l}< \\
0.001\end{array}$ & $<0.001$ & 1.000 \\
\hline SV (ml) & $\begin{array}{l}113.24 \pm \\
41.70\end{array}$ & $\begin{array}{l}95.75 \pm \\
37.42\end{array}$ & $\begin{array}{l}82.02 \pm \\
33.44\end{array}$ & $\begin{array}{l}79.91 \pm \\
32.62\end{array}$ & $\begin{array}{l}< \\
0.001\end{array}$ & $<0.001$ & 0.899 \\
\hline $\mathrm{CO}(\mathrm{L} / \mathrm{min})$ & $\begin{array}{l}7.49 \pm \\
2.38\end{array}$ & $\begin{array}{l}6.71 \pm \\
1.95\end{array}$ & $\begin{array}{l}6.14 \pm \\
1.84\end{array}$ & $\begin{array}{l}6.00 \pm \\
1.86\end{array}$ & 0.010 & $<0.001$ & 0.248 \\
\hline $\begin{array}{l}\text { SVR } \\
(\mathrm{mmHg} \cdot \mathrm{min} / \mathrm{L})\end{array}$ & $\begin{array}{l}0.82 \pm \\
0.20\end{array}$ & $\begin{array}{l}0.78 \pm \\
0.24\end{array}$ & $\begin{array}{l}0.81 \pm \\
0.26\end{array}$ & $\begin{array}{l}0.82 \pm \\
0.26\end{array}$ & 0.931 & 0.282 & 0.261 \\
\hline
\end{tabular}

Haemodynamic parameters in the supine position and in the titled upright position were compared between the two groups according to age groups. In the supine position, the SBP was higher (141.94 \pm 22.60 vs. $128.14 \pm 20.71, p=0.039)$ while the HR was lower (64.43 \pm 8.71 vs. $72.59 \pm 9.42, p=0.004)$ in patients aged 60 years and older than in patients under 60 years old. However, DBP, MBP, SV, CO and SVR were not significantly different between the two groups. The HR was lower while the SV was higher per minute after tilting upright in patients aged 60 years and older than in patients under 60 years old (Figure $1 \mathrm{~A}$ and $1 \mathrm{~B})$. However, BP, $\mathrm{CO}$ and SVR were not significantly different between the two groups.

Patients 60 years and older had lower HRmax and higher SVmin in terms of extremum haemodynamic parameters after tilting upright. The absolute $(\triangle H R m a x=H R m a x-H R s u p i n e)$ and percentage $(\triangle \mathrm{HRmax} \%=(\mathrm{HRmax}-\mathrm{HR}$ supine$) / \mathrm{HRsupine})$ increases to HRmax and the absolute $(\triangle \mathrm{SVmin}=\mathrm{SVmin}-$ SVsupine) and percentage ( $\triangle S V \%=(S V m i n-S V$ supine $) / S V$ supine) decreases to $S V m i n$ in patients aged 60 and older were lower in terms of the extremum of haemodynamic parameters during the transition from supine to tilted upright (Fig. $2 A-C$ ). The time to SBPmin (seconds) $(93.39 \pm 52.11$ vs. $93.61 \pm 53.15, p=$ 0.989) and SBPmin, DBPmin, MBPmin, COmin and SVRmax were not significantly different between the two groups.

Rate of change of haemodynamic parameters in the upright position

Comparing the rates of changes of haemodynamic parameters per minute within 3 minutes of tilting upright, it was found that the rates of changes of haemodynamic parameters were the most significant at 
the first minute of tilting upright, but there was no significant difference at the second and the third minute after tilting upright (Table 2).

Table 2

Comparison of the rate of change of haemodynamic parameters in the titled upright position

\begin{tabular}{|c|c|c|c|c|c|c|}
\hline Variables & 1st minute & 2nd minute & 3rd minute & $\begin{array}{l}p \\
\text { value* }\end{array}$ & $p_{\text {value }}$ & $p_{\text {value }}$ \\
\hline $\mathrm{dSBP} / \mathrm{dt}(\mathrm{mmHg} / \mathrm{s})$ & $\begin{array}{l}-0.47 \pm \\
0.36\end{array}$ & $-0.02 \pm 0.19$ & $-0.06 \pm 0.20$ & $<.001$ & 0.231 & $<0.001$ \\
\hline $\mathrm{dDBP} / \mathrm{dt}(\mathrm{mmHg} / \mathrm{s})$ & $\begin{array}{l}-0.06 \pm \\
0.16\end{array}$ & $\begin{array}{l}-0.002 \pm \\
0.10\end{array}$ & $-0.03 \pm 0.10$ & 0.094 & 0.316 & 0.304 \\
\hline $\mathrm{dMBP} / \mathrm{dt}(\mathrm{mmHg} / \mathrm{s})$ & $\begin{array}{l}-0.20 \pm \\
0.25\end{array}$ & $\begin{array}{l}-0.002 \pm \\
0.13\end{array}$ & $-0.05 \pm 0.14$ & $<.001$ & 0.127 & 0.002 \\
\hline $\mathrm{dHR} / \mathrm{dt}(\mathrm{bpm} / \mathrm{s})$ & $0.17 \pm 0.16$ & $0.01 \pm 0.07$ & $0.001 \pm 0.10$ & $<.001$ & 0.565 & $<0.001$ \\
\hline $\mathrm{dSV} / \mathrm{dt}(\mathrm{ml} / \mathrm{s})$ & $\begin{array}{l}-0.74 \pm \\
0.39\end{array}$ & $-0.03 \pm 0.13$ & $-0.05 \pm 0.18$ & $<.001$ & 0.527 & $<0.001$ \\
\hline $\mathrm{dCO} / \mathrm{dt}(\mathrm{L} / \mathrm{min} / \mathrm{s})$ & $\begin{array}{l}-0.03 \pm \\
0.03\end{array}$ & $\begin{array}{l}-0.001 \pm \\
0.009\end{array}$ & $\begin{array}{l}-0.004 \pm \\
0.01\end{array}$ & $\begin{array}{l}<.001 \\
0.01\end{array}$ & 0.268 & $<0.001$ \\
\hline $\begin{array}{l}\mathrm{dSVR} / \mathrm{dt} \\
(\mathrm{mmH} \cdot \mathrm{min} / \mathrm{L} / \mathrm{s})\end{array}$ & $\begin{array}{l}0.002 \pm \\
0.003\end{array}$ & $\begin{array}{l}0.0002 \pm \\
0.001\end{array}$ & $\begin{array}{l}0.0005 \pm \\
0.003\end{array}$ & 0.007 & 0.619 & 0.081 \\
\hline
\end{tabular}

The rate of increase in HR and the rate of decline in SV in patients 60 years and older in the tilted upright position were significantly lower than those in patients under 60 years of age (Figure 3). However, there were no significant differences in the rate of decline in SBP, DBP, MBP, CO or in the rate of increase in SVR between the two groups.

Factors influencing blood pressure from supine to tilt upright position

Bivariate correlation analysis showed that age, HR, SV, CO, SVR and SBP were all positively linearly correlated. When age, HR, SV and SVR were included in linear regression, linear regression analysis found that the changes in HR, SV and SVR were related to the change in SBP and that SV and SVR had more significant effects on SBP (Table 3). 
Table 3

Factors influencing blood pressure from supine to tilted upright position

\begin{tabular}{|lllll|}
\hline Variables & B & Beta & $\boldsymbol{p}$ value & $\mathbf{9 5 \%}$ Cl for $\mathbf{B}$ \\
\hline Age & 0.139 & 0.141 & 0.3 & $-0.128-0.407$ \\
\hline HR & 0.522 & 0.311 & 0.025 & $0.068-0.976$ \\
\hline SV & 0.698 & 0.787 & $\nabla 0.001$ & $0.503-0.892$ \\
\hline SVR & 0.061 & 0.653 & $\nabla 0.001$ & $0.040-0.082$ \\
\hline
\end{tabular}

\section{Discussion}

Continuous noninvasive blood pressure measurement in the head-up tilt test enabled us to observe rapid changes in blood pressure from the supine to the tilted upright position and to study underlying haemodynamic mechanisms in $\mathrm{OH}$ patients ${ }^{16,17}$. A total of 45 typical $\mathrm{OH}$ patients (mean age $57.18 \pm$ 15.94) were enrolled in our retrospective study, including 36 males and 9 females. This study aimed to gain insight into the clinical features and haemodynamic mechanisms underlying short-term orthostatic blood pressure decline patterns and the factors affecting blood pressure regulation.

Impaired increase in SVR

In patients with $\mathrm{OH}$, we observed an impaired increase in SVR from the supine to the tilted upright position (shown in Table 1), and linear regression analysis showed that an increase in SVR was the main determinant of the maintenance of SBP from the supine to the tilted upright position (shown in Table 3).

Physiologically, during active standing, the muscles of the lower limbs and abdomen contract to compress the blood vessels of the lower limbs and abdominal cavity, which increases the blood return to the heart and maintains blood pressure. However, for the $\mathrm{OH}$ patients in the current study, the blood pressure decrease in the tilted upright position was based on a supply-demand mismatch between increasing $\mathrm{CO}$ and an impaired increase in SVR, with an even pronounced decrease possible ${ }^{1,5}$. In general, the aetiology of $\mathrm{OH}$ is diverse and divided into nonneurogenic and neurogenic causes ${ }^{3}$. Herein, different causes of $\mathrm{OH}$ had different mechanisms for the impairment of SVR increase ${ }^{18-20}$. In nonneurogenic $\mathrm{OH}$ patients, SVR did not increase because of intravascular hypovolemia and consequently decreased $\mathrm{CO}$, which was usually accompanied by compensatory tachycardia. In neurogenic $\mathrm{OH}$ patients, sympathetic noradrenergic failure resulted in an inadequate SVR increase in the tilted upright position. In addition, vasoactive and psychoactive drugs could influence SVR. Therefore, it was particularly important to identify and clarify the aetiology of $\mathrm{OH}$ for patient treatment and prognosis. A noninvasive head-up tilted test could be used as an important detection method for evaluation of autonomic function ${ }^{12,21}$. Based on the changing trend of HR and SVR, further autonomic function was able to be evaluated. Based on the important role of SVR in the maintenance of tilted upright blood 
pressure, the use of leg stretch socks and repeated upright tilt training may be effective in improving the symptoms of $\mathrm{OH}$ patients ${ }^{22-24}$.

Pattern of haemodynamic changes in the upright position

In typical $\mathrm{OH}$ patients after tilting upright, we compared the amplitude and rate of change of haemodynamic parameters at each minute after tilting upright (shown in Table 1 and Table 2). It was found that the amplitude and rate of change of haemodynamic parameters were the greatest and the fastest in the first minute after tilting upright and that the changes gradually slowed down in the following two minutes. This pattern of haemodynamic changes was distinct from initial $\mathrm{OH}$ and delayed $\mathrm{OH}^{25}$. Previous studies ${ }^{25,26}$ in healthy teenagers and young adult subjects using beat-to-beat measurement of SV with calculation of $\mathrm{CO}$ and SVR have established that $\mathrm{CO}$ actually increases with the onset of tilting upright, whereas SVR falls markedly. Herein, the initial fall in BP upon standing was due to a mismatch of this increase in $\mathrm{CO}$ and a decrease in SVR and did not occur or was far less pronounced in a passive change of posture. In delayed $\mathrm{OH}$ patients, a previous study ${ }^{25}$ showed that the SVR increase was significantly lower, whereas changes in $\mathrm{CO}$ were not different. These findings ${ }^{7,27}$ indicated that delayed $\mathrm{OH}$ was associated with an impaired SVR response, which can point towards impaired sympathetic vasoconstrictor function or the use of vasodilators.

The influence of age on SV and HR changes

In $\mathrm{OH}$ patients younger than 60 years of age, HR increased significantly while SV decreased significantly in the first 3 minutes after tilting upright, both in terms of rates, absolute values and percentage of changes, compared with $\mathrm{OH}$ patients older than 60 years of age and older.

The significant decrease in SV in patients 60 years of age and older was due to decreased venous sclerosis and reduced compliance in older patients, leading to reduced cardiac volume and subsequent significant decrease in SV. Two factors may be involved ${ }^{28,29}$. First, in older patients, more blood accumulated in the abdominal cavity due to a decrease in the ability of blood vessels to constrict in upright position.. On the other hand, in older patients, a decrease in myocardial compliance resulted in a greater need for cardiac preload to maintain left ventricle filling. Thus, with increasing age, myocardial compliance decreased, diastolic left ventricular filling was limited, and cardiac preload decreased. .This may be the most important reason for the significant decrease in SV in the upright position. In the case of increased heart rate, the relative shortening of diastolic period was more pronounced.

Although SV decreased significantly after standing, there was no corresponding increase in HR. This can be ascribed to diminished HR regulation with increasing age, which was further supported in this study by the decrease in the regulation of autonomic function in the elderly. In the mean time, the obvious fall in SV was not adequately compensated by a significant increase in SVR., All of these conditions indicated that orthostatic hypotension was intrinsically dependent on sympathetic vasomotor responses ${ }^{30,31}$. 
This study was conducted at a single centre, hence limiting its generalizability. Importantly, however, our study was truly retrospective of a real-world population of syncope or dizziness patients with typical $\mathrm{OH}$. In linear regression analysis, a parsimonious model was generated because of a small sample size; this may have an impact on the strength of the association of predictors and main adverse events. Largescale, multicentre, and prospective studies should be conducted in the future, and subgroup analyses should be performed in groups of typical $\mathrm{OH}$.

\section{Conclusion}

In patients with $\mathrm{OH}$, we observed no significant increase in SVR from the supine to the tilted upright position. Nevertheless, an increase in SVR had a significant impact on the maintenance of SBP. In addition, in $\mathrm{OH}$ patients younger than 60 years of age, $\mathrm{HR}$ increased significantly while SV decreased significantly after tilting upright compared with $\mathrm{OH}$ patients 60 years of age and older. This condition may be related to the regulation of autonomic nervous function and vascular compliance.

\section{Abbreviations}

Orthostatic hypotension $(\mathrm{OH})$

Systolic blood pressures (SBP)

Diastolic blood pressures (DBP)

Mean arterial pressure (MAP)

Heart rate (HR)

Inter-beat interval (IBI)

Stroke volume (SV)

Cardiac output (CO)

Systemic vascular resistance (SVR)

\section{Declarations}

\section{Ethics approval and consent to participate}

The study protocol conforms to the ethical guidelines of the Declaration of Helsinki and was approved by the Ethics Committee of the Beijing Tiantan Hospital. The methods were strictly carried out in according with the approved guidelines. Confirm informed consent of all subjects and, if subject is under 18 years of age, obtain informed consent from parent and/or legal guardian. 


\section{Consent for publication}

Not Applicable.

\section{Availability of data and material}

The datasets used and/or analysed during the current study available from the corresponding author on reasonable request.

\section{Competing interests}

The authors have no conflicts of interest to declare.

\section{Funding}

1. Space Station Human Space Medicine Project (HYZHXM01011)

2. State Key Lab of Space Medicine Fundamentals and Application Research Fund Project (SMFA16A02)

3. State Key Lab of Space Medicine Fundamentals and Application Research Funding for Open Projects (SMFA19K02)

\section{Authors' contributions}

Wang Jiayu and Liu Jiexin were responsible for the conception and design of the article, the implementation and feasibility analysis of the research, and the analysis and interpretation of the results. Wang Jiayu and Wu Yueyang were responsible for data collection of head-up tilt test and the analysis of hemodynamic characteristics. Wang Jiayu, Li Zhaoxia and Yang Xiaomeng were responsible for statistical processing and writing of the paper. Wang Yongjun, Zhao Xingquan, Wang Yilong, Andre Aubert, Wu Bin, Li Yinghui, Wang Linjie, Qu Lina were responsible for literature retrieval, technical operation, project implementation, fund application, etc. Liu Jiexin was responsible for the quality control, review, supervision and management of the article. All authors reviewed the manuscript.

\section{Acknowledgement}

The authors thank Dr Liu Wenjian and Dr.Liu Zhenjun for their contributions during the performance of this study.

\section{References}

1. Tanaka H, Sjoberg BJ, Thulesius O. Cardiac output and blood pressure during active and passive standing. Clinical physiology. 1996;16:157-170.10.1111/j.1475-097x.1996.tb00565.x.

2. Goldstein DS, Robertson D, Esler M, et al. Dysautonomias: clinical disorders of the autonomic nervous system. Annals of internal medicine. 2002;137:753-763.10.7326/0003-4819-137-9- 
$200211050-00011$.

3. Arnold AC, Raj SR. Orthostatic Hypotension: A Practical Approach to Investigation and Management. The Canadian journal of cardiology. 2017;33:1725-1728.10.1016/j.cjca.2017.05.007.

4. Sutton R. Clinical classification of syncope. Progress in cardiovascular diseases. 2013;55:339344.10.1016/j.pcad.2012.11.005.

5. Fedorowski A, Melander O. Syndromes of orthostatic intolerance: a hidden danger. Journal of internal medicine. 2013;273:322-335.10.1111/joim.12021.

6. Nwazue VC, Raj SR. Confounders of vasovagal syncope: orthostatic hypotension. Cardiology clinics. 2013;31:89-100.10.1016/j.ccl.2012.09.003.

7. Finucane C, O'Connell MD, Fan CW, et al. Age-related normative changes in phasic orthostatic blood pressure in a large population study: findings from The Irish Longitudinal Study on Ageing (TILDA). Circulation. 2014;130:1780-1789.10.1161/CIRCULATIONAHA.114.009831.

8. Finucane C, O'Connell MD, Donoghue O, et al. Impaired Orthostatic Blood Pressure Recovery Is Associated with Unexplained and Injurious Falls. Journal of the American Geriatrics Society. 2017;65:474-482.10.1111/jgs.14563.

9. Gray-Miceli D, Ratcliffe SJ, Thomasson A, et al. Clinical Risk Factors for Orthostatic Hypotension: Results Among Elderly Fallers in Long-Term Care. Journal of patient safety. 2020;16:e143e147.10.1097/PTS.0000000000000274.

10. Krediet CT, van Dijk N, Linzer M, et al. Management of vasovagal syncope: controlling or aborting faints by leg crossing and muscle tensing. Circulation. 2002;106:16841689.10.1161/01.cir.0000030939.12646.8f.

11. Williford NN, Ward CC, Olshansky B. Evaluation and Management of Syncope: Comparing the Guidelines of the American College of Cardiology/American Heart Association/Heart Rhythm Society and the European Society of Cardiology. The Journal of innovations in cardiac rhythm management. 2018;9:3457-3463.10.19102/icrm.2018.091208.

12. Brignole M, Moya A, de Lange FJ, et al. 2018 ESC Guidelines for the diagnosis and management of syncope. European heart journal. 2018;39:1883-1948.10.1093/eurheartj/ehy037.

13. Wesseling KH, Jansen JR, Settels JJ, et al. Computation of aortic flow from pressure in humans using a nonlinear, three-element model. Journal of applied physiology. 1993;74:25662573.10.1152/jappl.1993.74.5.2566.

14. Harms MP, Wesseling KH, Pott F, et al. Continuous stroke volume monitoring by modelling flow from non-invasive measurement of arterial pressure in humans under orthostatic stress. Clinical science. 1999;97:291-301

15. Jellema WT, Wesseling KH, Groeneveld AB, et al. Continuous cardiac output in septic shock by simulating a model of the aortic input impedance: a comparison with bolus injection thermodilution. Anesthesiology. 1999;90:1317-1328.10.1097/00000542-199905000-00016.

16. Freeman R, Wieling W, Axelrod FB, et al. Consensus statement on the definition of orthostatic hypotension, neurally mediated syncope and the postural tachycardia syndrome. Autonomic 
neuroscience: basic \& clinical. 2011;161:46-48.10.1016/j.autneu.2011.02.004.

17. Buszko K, Kujawski S, Newton JL, et al. Hemodynamic Response to the Head-Up Tilt Test in Patients With Syncope as a Predictor of the Test Outcome: A Meta-Analysis Approach. Frontiers in physiology. 2019;10:184.10.3389/fphys.2019.00184.

18. Deegan BM, O'Connor M, Donnelly $\mathrm{T}$, et al. Orthostatic hypotension: a new classification system. Europace: European pacing, arrhythmias, and cardiac electrophysiology : journal of the working groups on cardiac pacing, arrhythmias, and cardiac cellular electrophysiology of the European Society of Cardiology. 2007;9:937-941.10.1093/europace/eum177.

19. Cheshire WP, Jr. Clinical classification of orthostatic hypotensions. Clinical autonomic research: official journal of the Clinical Autonomic Research Society. 2017;27:133-135.10.1007/s10286-0170414-x.

20. Goldstein DS, Sharabi Y. Neurogenic orthostatic hypotension: a pathophysiological approach. Circulation. 2009;119:139-146.10.1161/CIRCULATIONAHA.108.805887.

21. Cheshire WP, Jr., Goldstein DS. Autonomic uprising: the tilt table test in autonomic medicine. Clinical autonomic research: official journal of the Clinical Autonomic Research Society. 2019;29:215230.10.1007/s10286-019-00598-9.

22. Ricci F, De Caterina R, Fedorowski A. Orthostatic Hypotension: Epidemiology, Prognosis, and Treatment. Journal of the American College of Cardiology. 2015;66:848860.10.1016/j.jacc.2015.06.1084.

23. Fanciulli A, Goebel G, Metzler B, et al. Elastic Abdominal Binders Attenuate Orthostatic Hypotension in Parkinson's Disease. Movement disorders clinical practice. 2016;3:156-160.10.1002/mdc3.12270.

24. Canney M, O'Connell MD, Murphy CM, et al. Single Agent Antihypertensive Therapy and Orthostatic Blood Pressure Behaviour in Older Adults Using Beat-to-Beat Measurements: The Irish Longitudinal Study on Ageing. PloS one. 2016;11:e0146156.10.1371/journal.pone.0146156.

25. van Wijnen VK, Hove DT, Finucane C, et al. Hemodynamic Mechanisms Underlying Initial Orthostatic Hypotension, Delayed Recovery and Orthostatic Hypotension. Journal of the American Medical Directors Association. 2018;19:786-792.10.1016/j.jamda.2018.05.031.

26. van Wijnen VK, Harms MP, Go-Schon IK, et al. Initial orthostatic hypotension in teenagers and young adults. Clinical autonomic research: official journal of the Clinical Autonomic Research Society. 2016;26:441-449.10.1007/s10286-016-0382-6.

27. van Wijnen VK, Finucane $C$, Harms MPM, et al. Noninvasive beat-to-beat finger arterial pressure monitoring during orthostasis: a comprehensive review of normal and abnormal responses at different ages. Journal of internal medicine. 2017;282:468-483.10.1111/joim.12636.

28. Verheyden B, Gisolf J, Beckers F, et al. Impact of age on the vasovagal response provoked by sublingual nitroglycerine in routine tilt testing. Clinical science. 2007;113:329-

337.10.1042/CS20070042.

29. Tsutsui $Y$, Sagawa S, Yamauchi K, et al. Cardiovascular responses to lower body negative pressure in the elderly: role of reduced leg compliance. Gerontology. 2002;48:133-139.10.1159/000052831. 
30. Gurel NZ, Carek AM, Inan OT, et al. Comparison of autonomic stress reactivity in young healthy versus aging subjects with heart disease. PloS one.

2019;14:e0216278.10.1371/journal.pone.0216278.

31. Bock JM, Hughes WE, Casey DP. Age-Associated Differences in Central Artery Responsiveness to Sympathoexcitatory Stimuli. American journal of hypertension. 2019;32:564-

569.10.1093/ajh/hpz035.

\section{Figures}
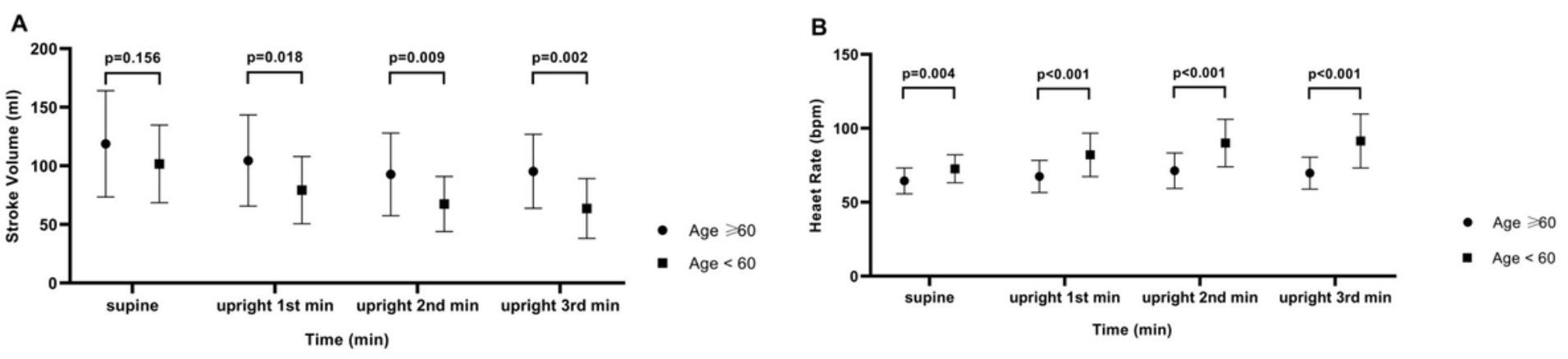

\section{Figure 1}

Comparison of SV and HR in the supine and tilted upright positions between the two groups The change of SV from the supine position to the tiled upright position per minute between two age groups is shown in (A). The change of HR from the supine position to the tiled upright position per minute between two age groups is shown in (B).

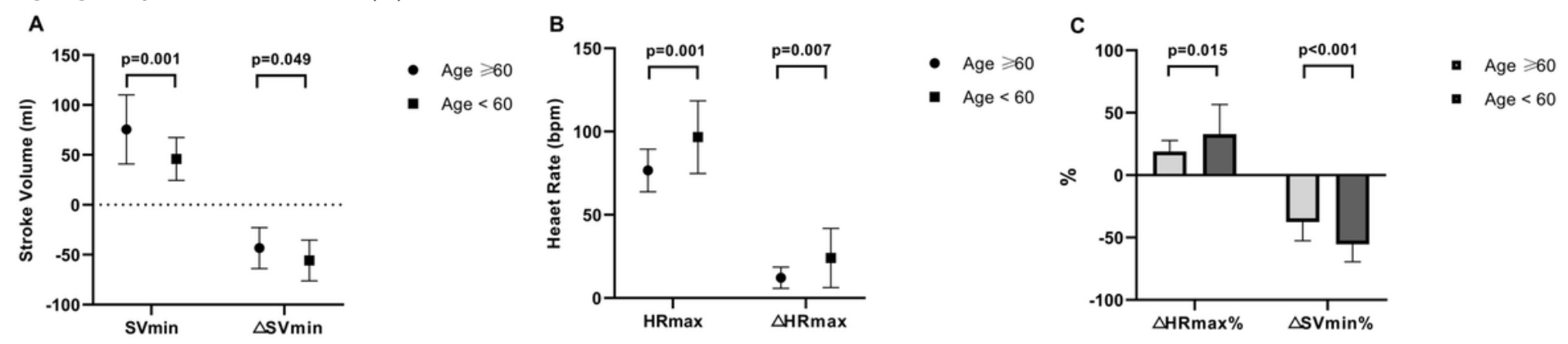

\section{Figure 2}

Comparison of the absolute value and percentage of the changes in SV and HR between the two groups The minimum value of SV after the tilted upright position and the absolute value of change of SV from the supine position to the tilted upright position between two groups is shown in (A). The maximum value of HR after the tilted upright position and the absolute value of change of HR from the supine position to the tilted upright position between two groups are shown in (B). The percentages of change of HR and SV from the supine position to the tilted upright postion between two groups are shown in (C). 

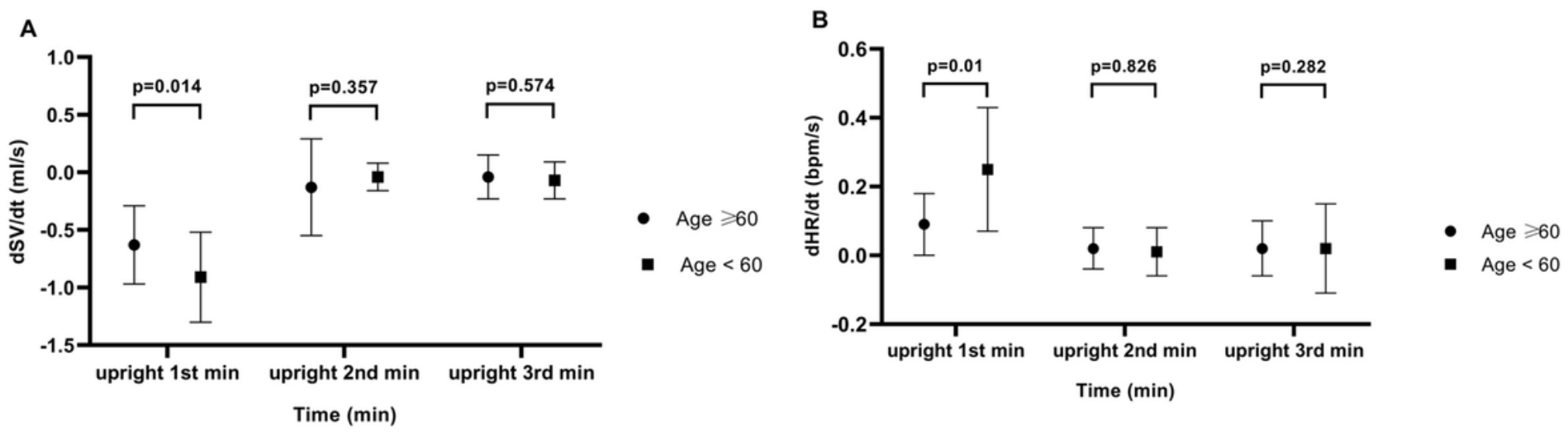

Figure 3

Comparison of the rate of change in SV and HR in the tilted upright position between the two groups The rate of change of SV from the supine position to the tiled upright position per minute between two age groups is shown in (A). The rate of change of HR from the supine position to the tiled upright position per minute between two age groups is shown in (B). 